\title{
Special Issue: IAMG 2019
}

\author{
Juliana Y. Leung ${ }^{1}$ - Liangping $\mathbf{L i}^{2}$. \\ ${\text { Eugene } \text { Morgan }^{3} \text {. Hamid Emami-Meybodi }}^{3}$
}

Published online: 4 November 2020

(C) International Association for Mathematical Geosciences 2020

IAMG 2019 was held at State College, PA, USA in August 2019. The conference was a great success and covered a variety of topics including geostatistics, geoinformatics, and geomodeling. The main theme of the conference was geomodeling issues at the intersection of food, water, and energy. This special issue features five selected papers from the conference, and other papers from the conference will appear in future issues as well.

Biot's equations for poroelasticity are important for modeling rock mechanics and coupling geomechanics with fluid flow. While these equations can be solved analytically for simple models, more complex models require numerical approximation. The first paper, titled Finite Element Solvers for Biot's Poroelasticity Equations in Porous Media, by Teeratorn Kadeethum, Sanghyun Lee, and Hamid M. Nick provides critical insight and guidance in the selection of finite element solvers for Biot's equations. By comparing five methods on various performance metrics, the results of the paper allow

\footnotetext{
Juliana Y. Leung

juliana2@ualberta.ca

Liangping Li

Liangping.Li@sdsmt.edu

Eugene Morgan

eum19@psu.edu

Hamid Emami-Meybodi

emami@psu.edu

1 University of Alberta, Edmonton, AB, Canada

2 South Dakota School of Mines and Technology, Rapid City, SD, USA

3 Pennsylvania State University, State College, PA, USA
} 
the practitioner to optimally select the appropriate solver. The paper also compares important properties of the solvers, including identifying those that conserve local mass and which require more degrees of freedom.

The second paper, The Physical Meaning of the Koval Factor by Jose Julian Salazar and Larry W. Lake, revisits the well-known Koval theory, which has long been used by the petroleum engineering community to analyze miscible displacement processes, particularly those encountered in enhanced oil recovery applications. The authors present a mathematical framework to better explain the physical meaning and significance of this theory. A large amount of core and petrophysical data is examined, and it is shown that the Koval factor captures the combined influence of mobility ratio and heterogeneity. This new framework may offer additional insights for the analysis of core flood results.

The nature and characteristics of geoscience data should be considered when they are used in statistical learning algorithms. In the third paper, titled Towards Geostatistical Learning for the Geosciences: A Case Study in Improving the Spatial Awareness of Spectral Clustering, Hassan Talebi, L.J.M. Peeters, Ute Mueller, Raimon Tolosana-Delgado, and K.G. van den Boogaart review several characteristics of geoscience data and challenges for statistical learning algorithms. They introduce a novel spatial-spectral clustering to improve the physical realism and spatial awareness of spectral clustering and to illustrate how statistical learners can be adapted for modeling geoscience data. This spatial-spectral clustering approach allows the use of existing domain knowledge to select the input parameters (e.g. geometry of the patterns, the order of spatial statistics, and weights for the convex combination of distance matrices). Using a case study, the authors illustrate how classical machine learning algorithms can be amended to account for the particularities of spatial data.

In the fourth paper, titled Multivariate Outlier Detection in Applied Data Analysis: Global, Local, Compositional and Cellwise Outliers, Peter Filzmoser and Mariella Gregorich present an overview of different techniques for multivariate outlier detection using data frequently found in the discipline of geosciences. In particular, they explore three common types of data in geological studies-spatial, compositional, and flat data - and illustrate how these types of data can be analyzed using existing outlier detection methodology.

Last but not least, the final paper, Compositional Data in Geostatistics: A Log-Ratio Based Framework to Analyze Regionalized Compositions by Vera Pawlowsky-Glahn and Juan José Egozcue, is an important contribution to the field of multivariate analysis for compositional data in the context of geospatial processes. The authors start with a review of regionalized compositions, and then a solution based on the principle of working in coordinates using orthonormal log-ratio representations is presented. The proposed method is illustrated using a case study of arsenic contamination in groundwater in Bangladesh.

We hope that you enjoy this collection of papers and that they inspire new research ideas. 